
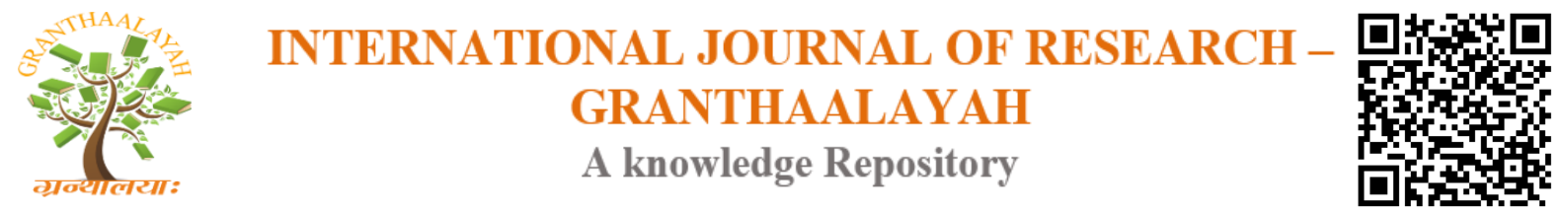

Management

\title{
PREDICTING BANKRUPTCY OF SELECTED MANUFACTURING COMPANIES LISTED IN COLOMBO STOCK EXCHANGE: APPLYING ALTMAN'S Z-SCORE
}

\author{
Anandasayanan.S ${ }^{* 1}$, Subramaniam V.A ${ }^{2}$ \\ ${ }^{* 1,2}$ Senior Lecturer, Department of Financial Management, University of Jaffna, Sri Lanka
}

DOI: https://doi.org/10.29121/granthaalayah.v5.i2.2017.1743

\begin{abstract}
Bankruptcy is the legal status for an individual or company incapable to pay off outstanding debt. Predication of Bankruptcy is critical task. Early stage of identification of likelihood of solvency may avoid evils in the near future \& may shelter the firm from Bankruptcy situation. Bankruptcy of organizations can be predicated by using Altman's Z-Score Model. This study tries to apply the model to understand the likelihood of Bankruptcy of selected listed manufacturing firms for past 5 years from 2010 to 2014 which are listed in Colombo Stock Exchange. The study reveals that four companies completely belong to Safe Zone for the entire period of study. Three firms are in Distress Zone which clearly indicates that these firms may go Bankrupt in near future.
\end{abstract}

Keywords: Bankruptcy; Manufacturing Companies; Z-Score Model.

Cite This Article: Anandasayanan.S, and Subramaniam V.A. (2017). "PREDICTING BANKRUPTCY OF SELECTED MANUFACTURING COMPANIES LISTED IN COLOMBO STOCK EXCHANGE: APPLYING ALTMAN'S Z-SCORE.” International Journal of Research - Granthaalayah, 5(2), 313-321. https://doi.org/10.29121/granthaalayah.v5.i2.2017.1743.

\section{Introduction}

Most of the organizations survive with an objective of profit maximization. To achieve profit maximization objective, firm needs strong internal \& external support. The failure of internal support system such as effective utilization of funds, labor, material etc \& external support system such as economic, political \& socio-cultural conditions results in Bankruptcy of the organization.

Bankruptcy is a situation where the firm's total liabilities exceed total assets. The real net worth of the firm is, therefore negative. This leads to reduced sales, increased cost \& losses, ineffective competition etc. Ultimately firm will be under distress stage. Under such situations it becomes difficult for investors \& lenders to analyze the financial performance of the organization. Several 
bankruptcy models for example, logit analysis, recursive portioning algorithm and neural networks are available but still Altman's model is considered to be superior and pervasively applied by researchers all over the world in the present days. Altman's Z-Score Model is the output of a credit-strength test that predicts company's likelihood of bankruptcy.

\section{Objectives of the Study}

This study intends to estimate likelihood of Bankruptcy of selected Listed Manufacturing firms by applying Altman's Z-Score Model.

\section{Altman's Z-Score Model}

\section{The Standard Z-Score}

The formula for the Z-Score (which incorporates those seven simple pieces of data) is:

Z-Score $=([$ Working Capital $/$ Total Assets $] \times$ 1.2 $)+([$ Retained Earnings $/$ Total Assets $] \mathbf{x}$ 1.4) + ([Operating Earnings / Total Assets] x 3.3) + ([Market Capitalization / Total Liabilities] x 0.6) + ([Sales / Total Assets] x 1.0)

In general, the lower the score, the higher the chance of bankruptcy. For example, a Z-Score above indicates financial soundness; below 1.8 suggests a high likelihood of bankruptcy. Altman added a statistical technique called multivariate analysis to the mix of traditional ratio analysis techniques, and this allowed him to consider not only the effects of several ratios on the "predictiveness" of his bankruptcy model, but to consider how those ratios affected each other's usefulness in the model. The model formed by Altman for predicting a company's financial health is as follows;

\section{The Original Z-Score Formula or for Manufacturing Firms}

$\mathrm{X} 1=$ Working Capital / Total Assets

X2 = Retained Earnings / Total Assets

X3 = Earnings Before Interest and Taxes / Total Assets

X4 = Market Value of Equity / Total Liabilities

X5 = Sales / Total Assets

$\mathrm{Z}$ score bankruptcy model:

$\mathrm{Z}=1.2 \mathrm{X} 1+1.4 \mathrm{X} 2+3.3 \mathrm{X} 3+0.6 \mathrm{X} 4+.999 \times 5$

Zones of Discrimination:

$Z>2.99$-"Safe" Zone

$1.81<Z<2.99$-"Gray" Zone

$\mathrm{Z}<1.81$-"Distress" Zone 


\section{Methodology}

\subsection{Data Collection \& Research Sample}

The study depends on the secondary source \& annual reports (financial statements) are collected from the websites of respective organizations. The time frame of data being collected is set for past 5 years i.e. from 2010 to 2014. Study covers the sample size of 19 companies which listed in Colombo Stock Exchange in Sri Lanka. Below shown are the companies which are selected for the study for the period of 5 years.

\begin{tabular}{|c|c|c|c|c|c|c|c|}
\hline Company Names & year & $\mathrm{X1}$ & $\mathrm{X} 2$ & $\mathbf{X 3}$ & $\mathrm{X4}$ & $\mathrm{X5}$ & ALTMANS \\
\hline \multirow[t]{5}{*}{ ACL Plastic Plc } & 2010 & 0.42035781 & 0.175055074 & 0.177502377 & 2.256600842 & 1.130651027 & 3.819875852 \\
\hline & 2011 & 0.42867228 & 0.204725561 & 0.084002859 & 1.902872582 & 1.295530453 & 3.515485957 \\
\hline & 2012 & 0.492250242 & 0.263112092 & 0.056400793 & 2.241472157 & 1.436170746 & 3.926233876 \\
\hline & 2013 & 0.461607018 & 0.283509472 & 0.107941336 & 1.10578635 & 1.210224395 & 3.180744296 \\
\hline & 2014 & 0.518273383 & 0.375780523 & 0.125045255 & 2.445490472 & 1.262500457 & 4.290464875 \\
\hline \multirow[t]{5}{*}{ Tokyo Cement } & 2010 & -0.064978507 & 0.289972969 & 0.100369877 & 0.424627315 & 0.843142638 & 1.757127567 \\
\hline & 2011 & -0.079606639 & 0.301970302 & 0.093300571 & 0.224467327 & 0.980377529 & 1.750180265 \\
\hline & 2012 & -0.127235293 & 0.30205712 & 0.06457695 & 0.212484614 & 0.987547296 & 1.598339614 \\
\hline & 2013 & -0.035343849 & 0.273636563 & 0.083262822 & 1.659045437 & 0.959591886 & 2.57046503 \\
\hline & 2014 & -0.070054483 & 0.286511672 & 0.048171807 & 1.60121799 & 0.983733154 & 2.420481872 \\
\hline \multirow{5}{*}{$\begin{array}{l}\text { SINGER } \\
\text { INDUSTRIES } \\
\text { (CEYLON) PLC }\end{array}$} & 2010 & 0.21967902 & 0.04464113 & 0.046837111 & 4.849771514 & 0.726491354 & 4.117029134 \\
\hline & 2011 & 0.191350228 & 0.042633892 & 0.036352487 & 3.011182669 & 0.686955636 & 2.902936167 \\
\hline & 2012 & 0.211468656 & 0.310526747 & 0.01885385 & 2.63980377 & 0.545441245 & 2.880041044 \\
\hline & 2013 & 0.194164349 & 0.29151633 & 0.007798928 & 3.913960296 & 0.603653523 & 3.618886242 \\
\hline & 2014 & 0.204631558 & 0.295310389 & -0.004144527 & 3.837268147 & 0.630192841 & 3.577869202 \\
\hline \multirow{5}{*}{$\begin{array}{l}\text { HAYLEYS } \\
\text { EXPORTS PLC }\end{array}$} & 2010 & 0.067927771 & 0.111075908 & -0.053694367 & 1.607273085 & 1.245731526 & 2.269923564 \\
\hline & 2011 & 0.110865513 & 0.068256693 & 0.004373334 & 0.938022398 & 0.906954638 & 1.712798064 \\
\hline & 2012 & 0.2137446 & 0.113807263 & 0.03772571 & 1.622012017 & 0.738353042 & 2.251878785 \\
\hline & 2013 & 0.180761092 & 0.145668849 & 0.038401772 & 1.150816728 & 0.774441248 & 2.012506832 \\
\hline & 2014 & 0.138886763 & 0.121085267 & 0.010083513 & 1.07375143 & 0.760519546 & 1.774229485 \\
\hline \multirow{5}{*}{$\begin{array}{l}\text { Lanka Aluminium } \\
\text { Industries } \\
\text { Performance }\end{array}$} & 2010 & 0.162120617 & 0.229461873 & 0.035596135 & 2.451093103 & 0.97842079 & 3.08233526 \\
\hline & 2011 & 0.076356241 & 0.117543375 & 0.014077887 & 0.693514439 & 0.796722826 & 1.51547673 \\
\hline & 2012 & 0.093475671 & 0.113256525 & 0.010491614 & 0.624373428 & 0.700837437 & 1.38081376 \\
\hline & 2013 & 0.083614109 & 0.108150111 & 0.034055927 & 0.026710734 & 0.666179336 & 1.04633742 \\
\hline & 2014 & 0.11069698 & 0.146141559 & 0.082882467 & 0.051892691 & 0.706593638 & 1.348675952 \\
\hline \multirow{2}{*}{$\begin{array}{l}\text { Richard Pieris } \\
\text { Exports PLC }\end{array}$} & 2010 & 0.060293988 & 0 & 0.007108201 & 0.577216853 & 0.868089945 & 1.310229905 \\
\hline & 2011 & 0.076356241 & 0.117543375 & 0.014077887 & 0.693514439 & 0.796722826 & 1.51547673 \\
\hline
\end{tabular}


[Anandasayanan et. al., Vol.5 (Iss.2): February, 2017] ICV (Index Copernicus Value) 2015: 71.21
ISSN- 2350-0530(O), ISSN- 2394-3629(P) IF: 4.321 (CosmosImpactFactor), 2.532 (I2OR) InfoBase Index IBI Factor 3.86

\begin{tabular}{|c|c|c|c|c|c|c|c|}
\hline & 2012 & 0.093475671 & 0.113256525 & 0.010491614 & 0.624373428 & 0.700837437 & 1.38081376 \\
\hline & 2013 & 0.083614109 & 0.108150111 & 0.034055927 & 0.428665327 & 0.666179336 & 1.287510176 \\
\hline & 2014 & 0.11069698 & 0.146141559 & 0.082882467 & 0.832796179 & 0.706593638 & 1.817218044 \\
\hline \multirow{5}{*}{$\begin{array}{ll}\text { Lanka } & \text { Floortiles } \\
\text { PLC } & \\
\end{array}$} & 2010 & 0.19088399 & 0.070709232 & 0.228119343 & 7.725215329 & 0.964697908 & 6.68067465 \\
\hline & 2011 & 0.184608957 & 0.329243137 & 0.174172176 & 2.305269381 & 0.836840059 & 3.477241009 \\
\hline & 2012 & 0.182899142 & 0.320154185 & 0.125899277 & 1.522263755 & 0.81920841 & 2.815729105 \\
\hline & 2013 & 0.250867745 & 0.381951326 & 0.144366265 & 1.844578194 & 0.892473349 & 3.311402092 \\
\hline & 2014 & 0.307098915 & 0.45655731 & 0.177334837 & 2.949829966 & 0.889758596 & 4.252560471 \\
\hline \multirow{5}{*}{$\begin{array}{l}\text { Laxapana } \\
\text { Batteries PLC }\end{array}$} & 2010 & 0.322273074 & -0.013148727 & 0.037021621 & 3.957886377 & 0.774296364 & 3.639519011 \\
\hline & 2011 & 0.217606436 & 0 & -0.307200595 & 3.650755844 & 1.197510577 & 2.635329842 \\
\hline & 2012 & 0.0316101 & 0.025774002 & -0.22528834 & 3.859057244 & 0.618821721 & 2.264820269 \\
\hline & 2013 & 0.051472466 & 0.010591221 & 0.065508351 & 2.528367964 & 0.702502982 & 2.512295988 \\
\hline & 2014 & 0.270784289 & 0.026442898 & 0.187928367 & 1.668937563 & 1.011139371 & 2.994626723 \\
\hline \multirow{5}{*}{$\begin{array}{l}\text { Orient Garments } \\
\text { PLC }\end{array}$} & 2010 & 0.167020529 & 0.093781705 & 0.073910032 & 0.082784551 & 2.125344727 & 2.750637582 \\
\hline & 2011 & 0.169154235 & 0.231601723 & 0.036293833 & 0.085979289 & 1.947224316 & 2.645809031 \\
\hline & 2012 & 0.079928332 & 0.151388908 & 0.002855992 & 0.056475662 & 1.593442097 & 1.944610738 \\
\hline & 2013 & -1.440732421 & -0.018268793 & 2.390958839 & 1.518127229 & 13.08913543 & 20.13572072 \\
\hline & 2014 & -0.105752155 & -0.317284211 & 0.325242281 & 0.30053741 & 1.08676004 & 1.769281532 \\
\hline \multirow{5}{*}{$\begin{array}{l}\text { primal Glass } \\
\text { Ceylon PLC }\end{array}$} & 2010 & -0.034138689 & 0.087051298 & 0.089045331 & 2.736270342 & 0.62626461 & 2.642781798 \\
\hline & 2011 & -0.016855173 & 0.14406791 & 0.102323482 & 1.606256816 & 0.75253086 & 2.235421308 \\
\hline & 2012 & 0.022770269 & 0.199162204 & 0.10830629 & 1.664631084 & 0.773617398 & 2.435958214 \\
\hline & 2013 & 0.004174072 & 0.286305409 & 0.127592518 & 1.060978519 & 0.791661997 & 2.255140878 \\
\hline & 2014 & 0.069377179 & 0.303902787 & 0.078655756 & 1.907018455 & 0.895797227 & 2.80828881 \\
\hline \multirow[t]{5}{*}{ Printcare PLC } & 2010 & 0.103779126 & 0.121695543 & 0.11896523 & 4.498918467 & 1.133622174 & 4.520467225 \\
\hline & 2011 & 0.105988319 & 0.160796424 & 0.123621863 & 6.306006263 & 0.979567755 & 5.523424636 \\
\hline & 2012 & 0.101851295 & 0.432833924 & 0.10469186 & 6.269435189 & 0.786442109 & 5.621775409 \\
\hline & 2013 & 0.144188451 & 0.447021843 & 0.081682529 & 6.690629713 & 0.755980191 & 5.838767086 \\
\hline & 2014 & 0.148804092 & 0.442578888 & 0.100240695 & 7.059183896 & 0.754437848 & 6.118917833 \\
\hline \multirow{5}{*}{$\begin{array}{l}\text { Royal Ceremics } \\
\text { Lanka PLC }\end{array}$} & 2010 & 0.003421929 & 0.280674232 & 0.182314222 & 7.081998685 & 0.373063153 & 5.620949537 \\
\hline & 2011 & 0.000502357 & 0.436043568 & 0.241443537 & 3.898092472 & 0.264993644 & 4.011676623 \\
\hline & 2012 & 0.000116014 & 0.472737096 & 0.099734816 & 3.366105018 & 0.249270657 & 3.260029711 \\
\hline & 2013 & -0.035715448 & 0.399134075 & 0.051286402 & 1.404797266 & 0.185084751 & 1.713137404 \\
\hline & 2014 & -0.080153608 & 0.397555265 & 0.045569174 & 1.802861506 & 0.189973536 & 1.882461756 \\
\hline \multirow{4}{*}{$\begin{array}{ll}\text { Sierra } & \text { Cables } \\
\text { PLC } & \\
\end{array}$} & 2010 & 0.308578425 & 0.142927025 & 0.037513249 & 2.450808182 & 0.315971322 & 2.480641899 \\
\hline & 2011 & 0.519488566 & 0.146880682 & 0.051627794 & 1.29981423 & 0.929814682 & 2.709094175 \\
\hline & 2012 & 0.251937105 & 0.117012354 & 0.006010675 & 0.821567068 & 0.657080429 & 1.635997717 \\
\hline & 2013 & 0.152016834 & 0.034470263 & -0.129906513 & 0.900720421 & 0.785290435 & 1.127709765 \\
\hline
\end{tabular}


[Anandasayanan et. al., Vol.5 (Iss.2): February, 2017] ICV (Index Copernicus Value) 2015: 71.21
ISSN- 2350-0530(O), ISSN- 2394-3629(P) IF: 4.321 (CosmosImpactFactor), 2.532 (I2OR) InfoBase Index IBI Factor 3.86

\begin{tabular}{|c|c|c|c|c|c|c|c|}
\hline & 2014 & 0.221694785 & 0.099705813 & 0.138393148 & 1.572350633 & 1.128345598 & 2.934075247 \\
\hline \multirow{5}{*}{$\begin{array}{l}\text { Swadeshi } \\
\text { Industrial Works } \\
\text { PLC }\end{array}$} & 2010 & -0.117426382 & 0.225845655 & 0.060295317 & 0.002934615 & 2.148761795 & 2.524769369 \\
\hline & 2011 & -0089525842 & 0252262562 & 0081103255 & 0002652750 & 2368131467 & 2883100441 \\
\hline & 2012 & 20075472072 & 0257326499 & 0022432574 & 0002595062 & 2411352518 & 2756627662 \\
\hline & 2013 & -0.11386326 & 0.276287051 & 0.03067176 & 0.003861493 & 2.25763238 & 2.611332043 \\
\hline & 2014 & -0.090238569 & 0.173497796 & -0.069989278 & 0.002820332 & 2.361805816 & 2.267144028 \\
\hline \multirow{5}{*}{$\begin{array}{l}\text { Swisstek Ceylon } \\
\text { PLC }\end{array}$} & 2010 & 0.105691317 & -0.289128346 & -0.011640735 & 3.47885578 & 0.186990654 & 1.957939591 \\
\hline & 2011 & -0.149750401 & -0.252855994 & 0.003363463 & 1.75039648 & 0.206009866 & 0.733648309 \\
\hline & 2012 & -0.097588528 & -0.237031951 & 0.010431998 & 1.20191801 & 0.253416998 & 0.560042433 \\
\hline & 2013 & -0.053758524 & -0.1742298 & 0.057858457 & 1.673932712 & 0.346856032 & 1.233716618 \\
\hline & 2014 & -0.013558727 & -0.061374958 & 0.081008504 & 4.148292775 & 0.308467632 & 2.962575946 \\
\hline \multirow{5}{*}{$\begin{array}{l}\text { Central Industries } \\
\text { PLC }\end{array}$} & 2010 & 0.466571284 & 0.420465068 & 0.137717258 & 6.255666847 & 1.396032779 & 6.752436473 \\
\hline & 2011 & 0.421962827 & 0.436258712 & 0.136280259 & 2.808357268 & 1.508693697 & 4.760550503 \\
\hline & 2012 & 0.421266032 & 0.460754001 & 0.111097358 & 2.507461846 & 1.48030673 & 4.501979959 \\
\hline & 2013 & 0.435060197 & 0.45124882 & 0.071621543 & 1.880288344 & 1.271282774 & 3.789627457 \\
\hline & 2014 & 0.433197874 & 0.442913017 & 0.110046284 & 1.869258081 & 1.360185971 & 3.984809229 \\
\hline \multirow[t]{5}{*}{ Kelani Cables } & 2010 & 0.42928795 & 0.320488042 & 0.076995408 & 1.813647127 & 1.357657757 & 3.663759679 \\
\hline & 2011 & 0.512699347 & 0.406039334 & 0.141475162 & 1.63961997 & 1.527701033 & 4.162035331 \\
\hline & 2012 & 0.429065147 & 0.367728341 & 0.076429806 & 0.898317808 & 1.226850808 & 3.047757706 \\
\hline & 2013 & 0.464404964 & 0.402133623 & 0.083210986 & 1.138768388 & 1.345411253 & 3.42354157 \\
\hline & 2014 & 0.419158511 & 0.562619683 & 0.134176373 & 0.825631633 & 1.886646873 & 4.115465653 \\
\hline \multirow[t]{5}{*}{ Samson } & 2010 & 0.285098397 & 0.385166952 & -0.096303595 & 1.592418837 & 1.231920478 & 2.750921727 \\
\hline & 2011 & 0.316172232 & 0.243231567 & 0.030496259 & 1.522573291 & 1.641723561 & 3.375836063 \\
\hline & 2012 & 0.038486185 & 0.312891819 & 0.093816439 & 1.657421353 & 1.645739309 & 3.43401834 \\
\hline & 2013 & 0.371556813 & 0.353325252 & 0.09626453 & 1.637562044 & 1.45623685 & 3.696970554 \\
\hline & 2014 & 0.172069282 & 0.282706096 & 0.047616259 & 0.94901522 & 1.16238073 & 2.491195189 \\
\hline \multirow[t]{5}{*}{ Lanka Ceramic } & 2010 & 0.116406668 & -0.311221673 & 0.165346344 & 55.79339168 & 0.47581049 & 34.20146609 \\
\hline & 2011 & 0.104667137 & -0.254681047 & 0.165346344 & 27.13449104 & 0.518867561 & 17.11425222 \\
\hline & 2012 & -0.010337167 & -0.012481689 & 0.064349069 & 3.552980404 & 0.14158852 & 2.455849723 \\
\hline & 2013 & -0.002280164 & 0.03946417 & 0.052875254 & 5.36008453 & 0.166885265 & 3.609937965 \\
\hline & 2014 & 0.043055548 & 0.127704062 & 0.219749091 & 8.575421049 & 0.14368071 & 6.244557682 \\
\hline
\end{tabular}

5. Data Analysis \& Findings

Interpretation:

\begin{tabular}{|c|l|l|l|}
\hline Company Name & \multicolumn{1}{|c|}{ Year } & \multicolumn{1}{c|}{ Z Value } & \multicolumn{1}{c|}{ Zone } \\
\hline Acl Plastic PLC & 2010 & 3.819875852 & Safe \\
\hline & 2011 & 3.515485957 & Safe \\
\hline & 2012 & 3.926233876 & Safe \\
\hline
\end{tabular}


[Anandasayanan et. al., Vol.5 (Iss.2): February, 2017] ICV (Index Copernicus Value) 2015: 71.21

\begin{tabular}{|c|c|c|c|}
\hline & 2013 & 3.180744296 & Safe \\
\hline & 2014 & 4.290464875 & Safe \\
\hline \multirow[t]{5}{*}{ Tokyo Cement } & 2010 & 1.757127567 & Distress \\
\hline & 2011 & 1.750180265 & Distress \\
\hline & 2012 & 1.598339614 & Distress \\
\hline & 2013 & 2.57046503 & Gray \\
\hline & 2014 & 2.420481872 & Gray \\
\hline \multirow[t]{5}{*}{ Singer Industries (Ceylon) PLC } & 2010 & 4.117029134 & Safe \\
\hline & 2011 & 2.902936167 & Gray \\
\hline & 2012 & 2.880041044 & Gray \\
\hline & 2013 & 3.618886242 & Safe \\
\hline & 2014 & 3.577869202 & Safe \\
\hline \multirow[t]{5}{*}{ Hayleys Exports PLC } & 2010 & 2.269923564 & Gray \\
\hline & 2011 & 1.712798064 & Distress \\
\hline & 2012 & 2.251878785 & Gray \\
\hline & 2013 & 2.012506832 & Gray \\
\hline & 2014 & 1.774229485 & Distress \\
\hline \multirow[t]{5}{*}{$\begin{array}{l}\text { Lanka Aluminium Industries } \\
\text { Performance }\end{array}$} & 2010 & 3.08233526 & Safe \\
\hline & 2011 & 1.51547673 & Distress \\
\hline & 2012 & 1.38081376 & Distress \\
\hline & 2013 & 1.04633742 & Distress \\
\hline & 2014 & 1.348675952 & Distress \\
\hline \multirow[t]{5}{*}{ Richard Pieris ExportsPLC } & 2010 & 1.310229905 & Distress \\
\hline & 2011 & 1.51547673 & Distress \\
\hline & 2012 & 1.38081376 & Distress \\
\hline & 2013 & 1.287510176 & Distress \\
\hline & 2014 & 1.817218044 & Gray \\
\hline \multirow[t]{5}{*}{ Lanka Floortiles PLC } & 2010 & 6.68067465 & Safe \\
\hline & 2011 & 3.477241009 & Safe \\
\hline & 2012 & 2.815729105 & Gray \\
\hline & 2013 & 3.311402092 & Safe \\
\hline & 2014 & 4.252560471 & Safe \\
\hline \multirow[t]{5}{*}{ Laxapana Batteries PLC } & 2010 & 3.639519011 & Safe \\
\hline & 2011 & 2.635329842 & Gray \\
\hline & 2012 & 2.264820269 & Gray \\
\hline & 2013 & 2.512295988 & Gray \\
\hline & 2014 & 2.994626723 & Safe \\
\hline \multirow[t]{5}{*}{ Orient Garments PLC } & 2010 & 2.750637582 & Gray \\
\hline & 2011 & 2.645809031 & Gray \\
\hline & 2012 & 1.944610738 & Gray \\
\hline & 2013 & 20.13572072 & Safe \\
\hline & 2014 & 1.769281532 & Distress \\
\hline \multirow[t]{5}{*}{ Primal Glass Ceylon PLC } & 2010 & 2.642781798 & Gray \\
\hline & 2011 & 2.235421308 & Gray \\
\hline & 2012 & 2.435958214 & Gray \\
\hline & 2013 & 2.255140878 & Gray \\
\hline & 2014 & 2.80828881 & Gray \\
\hline
\end{tabular}




\begin{tabular}{|c|c|c|c|}
\hline \multirow[t]{5}{*}{ Print Care PLC } & 2010 & 4.520467225 & Safe \\
\hline & 2011 & 5.523424636 & Safe \\
\hline & 2012 & 5.621775409 & Safe \\
\hline & 2013 & 5.838767086 & Safe \\
\hline & 2014 & 6.118917833 & Safe \\
\hline \multirow[t]{5}{*}{ Royal Ceremics Lanka PLC } & 2010 & 5.620949537 & Safe \\
\hline & 2011 & 4.011676623 & Safe \\
\hline & 2012 & 3.260029711 & Safe \\
\hline & 2013 & 1.713137404 & Distress \\
\hline & 2014 & 1.882461756 & Gray \\
\hline \multirow[t]{5}{*}{ Sierra Cables PLC } & 2010 & 2.480641899 & Gray \\
\hline & 2011 & 2.709094175 & Gray \\
\hline & 2012 & 1.635997717 & Distress \\
\hline & 2013 & 1.127709765 & Distress \\
\hline & 2014 & 2.934075247 & Gray \\
\hline \multirow[t]{5}{*}{ Swadeshi Industrial Works PLC } & 2010 & 2.524769369 & Gray \\
\hline & 2011 & 2.883100441 & Gray \\
\hline & 2012 & 2.756627662 & Gray \\
\hline & 2013 & 2.611332043 & Gray \\
\hline & 2014 & 2.267144028 & Gray \\
\hline \multirow[t]{5}{*}{ Swisstek Ceylon PLC } & 2010 & 1.957939591 & Gray \\
\hline & 2011 & 0.733648309 & Distress \\
\hline & 2012 & 0.560042433 & Distress \\
\hline & 2013 & 1.233716618 & Distress \\
\hline & 2014 & 2.962575946 & Gray \\
\hline \multirow[t]{5}{*}{ Central Industries PLC } & 2010 & 6.752436473 & Safe \\
\hline & 2011 & 4.760550503 & Safe \\
\hline & 2012 & 4.501979959 & Safe \\
\hline & 2013 & 3.789627457 & Safe \\
\hline & 2014 & 3.984809229 & Safe \\
\hline \multirow[t]{5}{*}{ Kelani Cables PLC } & 2010 & 3.663759679 & Safe \\
\hline & 2011 & 4.162035331 & Safe \\
\hline & 2012 & 3.047757706 & Safe \\
\hline & 2013 & 3.42354157 & Safe \\
\hline & 2014 & 4.115465653 & Safe \\
\hline \multirow[t]{5}{*}{ Samson PLC } & 2010 & 2.750921727 & Gray \\
\hline & 2011 & 3.375836063 & Safe \\
\hline & 2012 & 3.43401834 & Safe \\
\hline & 2013 & 3.696970554 & Safe \\
\hline & 2014 & 2.491195189 & Gray \\
\hline \multirow[t]{5}{*}{ Lanka Ceramic PLC } & 2010 & 34.20146609 & Safe \\
\hline & 2011 & 17.11425222 & Safe \\
\hline & 2012 & 2.455849723 & Gray \\
\hline & 2013 & 3.609937965 & Safe \\
\hline & 2014 & 6.244557682 & Safe \\
\hline
\end{tabular}

Among the companies under study Acl Plastic PLC, Print Care PLC, Central Industries PLC and Kelani Cables are in the safe zone for the entire period of study. In the case of Lanka Floor Tiles 
PLC and Lanka Ceramic, even though they are in the Gray zone in the year 2012, prior and after that year they are in the safety zone. Singer Industries (Ceylon) PLC is identified under gray zone in the years 2011 and 2012, however, they shifted to safety zone in the years 2013 and 2014. Further Laxapana Batteries PLC has shifted to safety zone from Gray zone in the year 2014.In indicates that the above companies are in a good financial health condition and there are no symptoms for the bankruptcy in the near future. Thus, banks, financial companies, suppliers and other investors can continue their financial transactions with these companies.

Primal Glass Ceylon PLC and Swadeshi Industrial Works PLC are classified in Grey zone for the entire period of study and their scores are near to safety zone. Similarly, Tokyo Cement Company were in the distress zone from the year 2010 to 2012 and later improved their position to Gray zone in the years 2013 and 2014. Although, Royal Ceremics Lanka PLC was in the safety zone during the period from 2010 to 2012, it moved down to Grey zone in 2014. Samson Company was in the safety zone up to 2013 and shifted to grey in 2014. Similarly, Sierra Cables PLC and Swisstek Ceylon PLC also improved to Grey zone in the year 2014 and their scores for that year are near to safety zone. Richard Pieris Exports PLC was in the Distress zone up to 2013 and improved to Grey in 2014. According to the scores of these companies, there are chances for bankruptcy within the next 2 years of operations.

Lanka Aluminium Industries Performance Company was classified in the Distress zone in the entire period of study except in the year 2010.In the case of Hayleys Exports PLC, it was in the Grey zone in the years 2012 and 2013, however, it moved down to distress zone in the year 2014. Finally in the year 2014, Hayleys Exports PLC, Lanka Aluminium Industries Performance Company and Orient Garments PLC are in the distress zone. It implies that these companies are probably headed for bankruptcy in the very near future. It is a warning signal for the stake holders of the companies. Banks, financial companies and suppliers will refuse to continue further financial transactions with these companies in order to protect them.

\section{Conclusion}

This study attempts to estimate likelihood of Bankruptcy of selected Listed Manufacturing firms in Sri Lanka by applying Altman's Z-Score Model. It considers the data of 19 manufacturing companies, It was found that only four companies are identified under safety zone for the entire period of study. There are three companies in the distress zone at the end of the study period. These findings will support the stake holders of those companies to assess the likelihood of Bankruptcy of those companies and to determine their financial strategies.

\section{References}

[1] Diakomihalis, M. (2012). The accuracy of Altman's models in predicting hotel bankruptcy. International Journal of Accounting and Financial Reporting,

[2] Lakshan, A. M. I., \& Wijekoon, W. M. H. N. (2013). The use of Financial Ratios in Predicting Corporate Failure in Sri Lanka. GSTF Journal of Business Review (GBR), 2(4), 37-43.

[3] Niresh and Pratheepan, (2015). The Application of Altman's Z-Score Model in Predicting Bankruptcy: Evidence from the Trading Sector in Sri Lanka. International Journal of Business and Management; Vol. 10, No. 12 
[4] Carson M. J. (1995). Financial Distress in the Life Insurance Industry: An Empirical Examination. Illinois University.

[5] Altman, E. I. (1968). Financial Ratios, Discriminant Analysis and the Prediction of Corporate Bankruptcy. The Journal of Finance, 23(4), 589-609. http://dx.doi.org/10.1111/j.15406261.1968.tb00843.x

[6] Scott, J. (1981). The probability of bankruptcy: A comparison of empirical predictions and theoretical models. Journal of Banking \& Finance, 5, 318-344. http://dx.doi.org/10.1016/0378-4266(81)90029-7

[7] Sajjan (2016) PREDICTING BANKRUPTCY OF SELECTED FIRMS BY APPLYING ALTMAN'S Z-SCORE MODEL Http://www.granthaalayah.com OInternational Journal of Research GRANTHAALAYAH [152-158]

*Corresponding author.

E-mail address: sayananakshi@yahoo.com 